\title{
Influence of Experience on Postural Control: Effect of Expertise in Gymnastics
}

\author{
Geoffroy Gautier ${ }^{1}$, Régis Thouvarecq ${ }^{1}$, Jacques Larue ${ }^{2}$ \\ ${ }^{1}$ CETAPS, Faculté des Sciences du Sport, Université de Rouen, France. ${ }^{2}$ AMCO, Faculté du Sport et de l'Education Physique, \\ Université d'Orléans, France.
}

\begin{abstract}
The authors investigated how expertise in motor skills that require fine postural control, such as gymnastics, influences postural regulation. Gymnasts and nongymnasts performed a postural stabilization task after anterior-posterior destabilization while looking at a target in front of them. The authors recorded and analyzed the center of pressure and the ankle, knee, and hip displacements. Gymnasts were able to react rapidly after destabilization to decrease their center of pressure and the angular movements. Moreover, they used their knees to stabilize posture, whereas the nongymnasts used their hips. These findings suggest that specific postural experience modifies the ability to coordinate and regulate posture. The authors discuss these results from an ecological perspective.
\end{abstract}

Keywords: balance, expertise, gymnastics, humans, perception-action

E rect posture is a common position in daily life and a natural basis for the organization of the human-environment relationship (Duarte \& Zatsiorsky, 2000; Kluzik, Horak, \& Peterka, 2005; Sarre, 2000). Postural control is an active process that the nervous system engages to maintain upright balance. Studying this process experimentally or otherwise is a research paradigm (Clark \& Riley, 2007; Duarte \& Zatsiorsky; Horak \& Nashner, 1986; Kluzik et al; Mesure, Amblard, \& Cremieux, 1997; Nashner \& McCollum, 1985; Riccio \& Stoffregen, 1988; Riley \& Clark, 2003; Stoffregen \& Riccio, 1988; Stoffregen, Smart, Bardy, \& Pagulayan, 1999; Van der Kooij, Jacobs, Koopman, \& Grootenboer, 1999). Basing our ideas on an ecological approach, we suggest that an organism's possibilities for action (affordances) emerge from the interactions between the organism and its environment (Gibson, 1986; Riccio \& Stoffregen). Erect posture can be one of these affordances, and the same environment can have different affordances for different organisms. Posture itself also affords a great many other behaviors. Posture is not an end in itself, but rather it supports the accomplishment of suprapostural tasks (Stoffregen et al.). Affordances in postural control depend on constraints defined by the environment, the task, and the organism's properties (Bardy, Marin, Stoffregen, \& Bootsma, 1999; Riccio \& Stoffregen; Stoffregen \& Riccio). With regard to these three types of constraint, researchers have addressed the question of how the organism's properties influence postural control by studying the effects of biomechanical parameters (Bardy et al.) and aging or pathologies (Bronstein \& Guerraz, 1999; Horak, 2006; Sparrow \& Tirosh, 2005). Researchers have also explored the question from a developmental perspective (Lejeune et al., 2006) and by the investigation of the intense motor experience created, for example, by sports practice. Indeed, postural control is widely acknowledged to be influenced by sports experience, particularly the practice of sports requiring fine postural control (Asseman, Caron, \& Cremieux, 2004; Bringoux, Marin, Nougier, Barraud, \& Raphel, 2000; Era, Konttinen, Mehto, Saarela, \& Lyytinen, 1996; Golomer, Cremieux, Dupui, Isableu, \& Ohlmann, 1999; Marin, Bardy \& Bootsma, 1999; Mesure et al.; Perrin, Deviterne, Hugel, \& Perrot, 2002; Vuillerme, Danion, et al., 2001; Vuillerme \& Nougier, 2004; Vuillerme, Teasdale, \& Nougier, 2001). Most studies on this topic have focused on the effect of expertise in gymnastics because it clearly requires fine postural control, but the conclusions still diverge.

Some of these researchers have demonstrated an effect of gymnastics expertise on postural control abilities: Gymnasts were more sensitive to changes in body orientation and more accurate in estimating the subjective postural vertical than nongymnasts (Bringoux et al., 2000). Other results indicated that gymnasts more rapidly took advantage of the reinsertion of ankle proprioceptive information to decrease their center of pressure displacements (Vuillerme, Teasdale, \& Nougier, 2001). Moreover, expertise in gymnastics was shown to lead to functional modifications in the patterns of postural coordination (Marin et al., 1999). Gymnasts were able to defer the threshold of the transition from in-phase mode to antiphase mode of the ankle-hip relative phase. In contrast, other researchers were unable to validate the hypothesis of an effect of gymnastics expertise on postural control. Similar sways and visual dependence in erect posture were observed in gymnasts and nongymnasts (Vuillerme, Danion, et al., 2001; Vuillerme \& Nougier, 2004). Gymnastics expertise had an effect only when the participants had to assume a challenging posture (unipedal stance; Vuillerme, Danion, et al.; Vuillerme \& Nougier). Yet challenging postures such as the unipedal stance or the handstand are specific to the training of gymnasts, whereas nongymnasts are unable to reach similar level of performance because of a lack of training. Thus, the better performances of gymnasts are more likely due to the effects of skill learning and training in these challenging postures than to a better postural

Correspondence address: Geoffroy Gautier, Bd SiegfriedFaculté des Sciences du Sport, Université de Rouen, 76821 Mont Saint Aignan Cedex, France. E-mail address: geoffroy.gautier@etu .univ-rouen.fr 
control or a greater sensitivity to their body movements (Balter, Stokroos, Akkermans, \& Kingma, 2004). Moreover, according to Asseman et al. (2004), the postural ability of elite gymnasts in a specific posture is not transferable to upright standing posture.

Differences in experimental design can help researchers to explain many of the contradictions in the results and conclusions of these studies. All of the researchers who demonstrated an effect of gymnastics expertise on postural control analyzed the participants' activity during tasks in which the sensory-motor state had to change. In contrast, when the posture had to be maintained in a normal situation (i.e., without destabilization), the effect of gymnastics expertise was weaker. The point is that standing is a natural posture, is part of human development, has been thoroughly learned by most, and is widely assumed in the tasks of daily life (Duarte \& Zatsiorsky, 2000; Kluzik et al., 2005; Sarre, 2000). Therefore, every individual-gymnast or not—is an expert of erect posture, and researchers must expect a ceiling effect of the influence of expertise. The choice of variables and how they are measured can be important too. Using nonlinear time series measures of sway, Schmit, Regis, and Riley (2005) showed that no quantitative difference in postural control existed between ballet dancers and runners and that instead there were qualitative differences in the temporal and spatial structure of their postural stability. This finding suggests that the effect of expertise may be characterized more by properties of the postural balance evolution than by the quantity of movement. Nevertheless, most researchers accept the classical assumption that less motion corresponds to better postural performance (Asseman et al., 2004; Balter et al., 2004; Vuillerme, Danion, et al., 2001; Vuillerme \& Nougier, 2004; Vuillerme, Teasdale, et al., 2001). This assumption creates an important problem of conception. It seems more appropriate to think about postural movements as emergent phenomena that depend on interactions between the environment constraints, the task, and the organism (Bardy et al., 1999; Riccio \& Stoffregen, 1988; Stoffregen \& Riccio, 1988). For example, successful focusing on a distant target allows more postural sway than does focusing on a nearby target. Thus, there is some ambiguity in the relation between quantity of movement and postural performance (Stoffregen et al., 1999). From this point of view, better control does not necessarily correspond to a reduction of sway, but it must be defined according to the constraints to which the posture is subjected.

Therefore, we hypothesized that qualitative and quantitative differences in postural control would occur with differences in experience and that these differences would manifest as modifications in postural stability. From this perspective, we agreed with Riccio and Stoffregen (1988) that a stable postural state corresponds to a minimization of uncontrolled movements in the perception-action coupling when destabilization occurs. Uncontrolled movements indicate the loss of stability, which hinders the successful performance of suprapostural tasks.
By provoking postural destabilization and then observing the recovery of the postural stable state, we expected to determine the influence of gymnastics expertise on postural control. We assumed that the gymnasts had a better sensitivity to movements than the nongymnasts (Bringoux et al., 2000; Vuillerme, Teasdale, et al., 2001), and that their experience had led to functional modifications in postural coordination (Marin et al., 1999). Therefore, we specifically hypothesized that the gymnasts would-according to the constraints of the environment, the organism, and the task-(a) display a difference in their sensitivity to destabilization in comparison with the nongymnasts and (b) recover a stable postural state with a postural pattern characterized by specific spatial and temporal parameters. We predicted that the gymnasts would react more quickly to a postural perturbation and adopt a qualitatively different postural pattern adapted to the constraints.

\section{Method}

\section{Participants}

Two groups of athletes gave their informed consent to participate in this experiment. None of the athletes presented postural problems. As suggested by Vuillerme, Teasdale, et al. (2001), we constituted the two groups to differentiate our findings on sports experience requiring fine postural control from the impact of sports practice in general. A group of expert gymnasts comprised 8 nationally-ranked-men $(M=20.4, S D=2.2)$. The control group comprised 8 nongymnasts $(M=22.3, S D=2.4)$ who were all experts in other sports (handball, sprinting, and football; see Table 1).

\section{Procedure}

The experiment consisted of a sequence of three trials in which we asked the participants to stand barefoot on a force plate for $30 \mathrm{~s}$ while they looked at a target $(2 \times 2 \mathrm{~cm})$ that we had placed $2 \mathrm{~m}$ in front of their eyes. During the first $5 \mathrm{~s}$, they maintained their balance with a weight that was fixed behind them. The weight represented $7 \%$ of their body

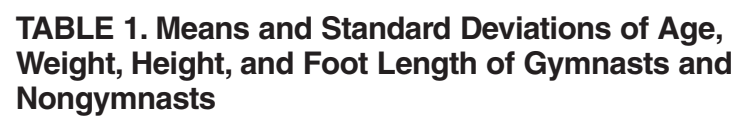

TABLE 1. Means and Standard Deviations of Age, Weight, Height, and Foot Length of Gymnasts and Nongymnasts

\begin{tabular}{lrrrrr}
\hline \hline \multirow{2}{*}{ Variable } & \multicolumn{2}{c}{ Gymnast } & & \multicolumn{2}{c}{ Nongymnast } \\
\cline { 2 - 3 } \cline { 6 - 7 } & \multicolumn{1}{c}{$M$} & $S D$ & & \multicolumn{1}{c}{$M$} & $S D$ \\
\hline Age (years) & 20.4 & 2.2 & & 22.3 & 2.4 \\
Weight (kg) & 68.5 & 5.6 & & 70.3 & 6.4 \\
Height (cm) & 171.3 & 6.8 & & 173.2 & 7.5 \\
Foot length (cm) & 26.4 & 1.2 & & 26.4 & 1.1 \\
\hline
\end{tabular}

Note. No significant difference appeared between the two groups. For both groups, $n=8$. 
mass, and the height of the weight support was adapted to each participant's height to ensure the same anteriorposterior constraint parallel to the ground (see Figure 1). At the 5th s, we removed the weight, and the participants had to stabilize and maintain their balance for the following 25 $\mathrm{s}$. The participants were not informed of when the trial actually began and when the weight would be removed.

We used a QFP systems force plate $(46 \times 46 \mathrm{~cm})$ with three strain gauges linked to a computer (Winposture version 1.27 software, PODO Technology, Atlanta, GA) to record the center of pressure (COP) position on the anteriorposterior axis $(y)$ throughout the trials $(40 \mathrm{~Hz})$. The participants' foot positioning was free but was recorded from trial to trial for each participant.

When an individual's posture is perturbed, the individual then reduces the uncontrolled movements caused by the perturbation to recover a stable postural state (Horak \& Nashner, 1986; Kluzik et al., 2005; Riccio \& Stoffregen, 1988). We thus divided each trial into four parts according to the postural activity. During Phase 1, the participants stood in a stable posture with the weight fixed behind them. Phase 2 (destabilization) began when the weight was removed, and finished when the peak COP magnitude in the anterior-posterior displacement was reached. Phase 3 (restabilization) began at the first acquisition following the peak of magnitude, with the limit between Phases 3 and 4 defined by the moment when the COP data in the anterior-posterior axis stayed within $2 S D$ of the data of the 30-s trials in a stable postural state (Kluzik et al.). Then Phase 4 (stability) began (see Figure 1).

We determined four dependent variables concerning the COP displacements. The first was the mean sway latencies (SLs) determined by the time elapsed between the 5th $\mathrm{s}$ (weight removed) and the return to a stable postural state (i.e., the time elapsed from the beginning of Phase 2 to the end of Phase 3 ). The second variable was the proportion (in percentage) of the SL devoted to the destabilization and restabilization phases, respectively. The third variable was the maximal magnitude of the COP displacement during the SL phase. Last, we compared the standard error (SE) of the means of the COP positions during Phases 2 and 3 with those of the stable postural state and the SE of the two groups during the destabilization and restabilization phases. To ensure that the initial conditions and the influence of the weight were similar for the two groups, we compared the postural activity with the weight in quiet stance during three 30 -s trials. All trials (with or without perturbation) were presented in random order.

Moreover, we videotaped the entire performance during all trials. A profile view of the participants was obtained from a camera placed $6 \mathrm{~m}$ from the participant and $1.4 \mathrm{~m}$ from the ground. Before video acquisition, five reference markers were fixed on the right side of the participants, one each on the small toe, ankle, knee, hip, and shoulder. The markers were used to identify the movements of the ankles, knees, and hips in the sagittal plane. Software developed with Matlab R12 was used for the video analysis, which consisted of sampling the video at $50 \mathrm{~Hz}$. The dependent variable was the SE of each angle to determine the size of each angle variation in the SL period until recovery of a postural stable state (Phases 2, 3, and 4; see Figure 1) and the maximal angle variation magnitude (MAVM) of each cited joint during each phase. MAVM resulted from the subtraction of the minimal joint angle from the maximal angle.

The distributions of data respected the criteria of normality (K-S and Lilliefors test), and therefore we applied $t$ tests for pairwise comparisons of the data (initial condition,

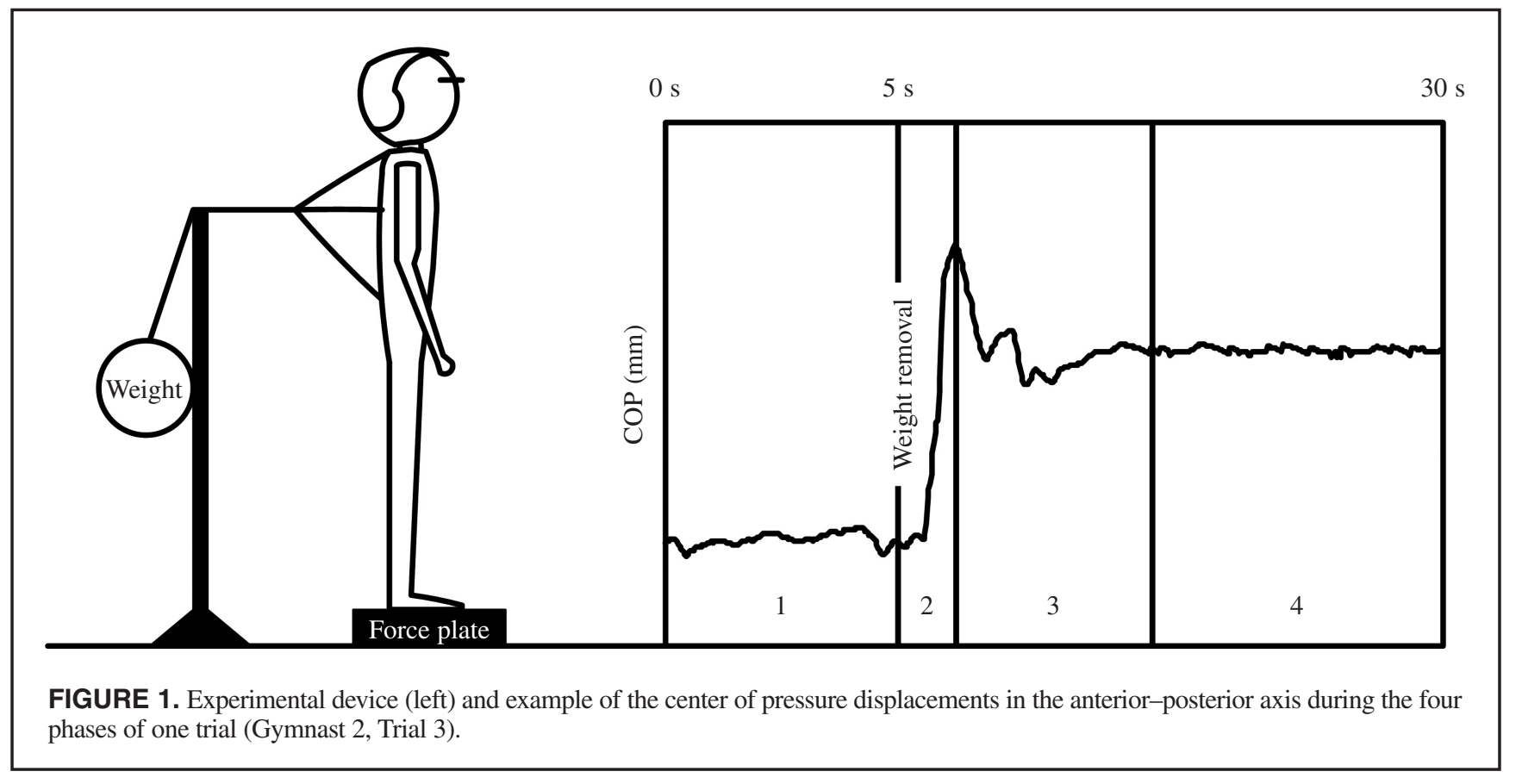


SLs, and magnitude of the COP). A 2 (groups) $\times 3$ (phases) ANOVA with repeated measures on the second factor was applied to analyze the SE and MAVM data. Finally, pairwise comparisons were made between mean values by using Tukey's post hoc tests. The threshold for significant differences was set at $p=.05$.

\section{Results}

\section{Initial Conditions}

A $t$ test showed no significant difference for either velocity, $t(14)=0.07$ (gymnast: $M=14.33 \mathrm{~mm} / \mathrm{s}, S D=8.33$ $\mathrm{mm} / \mathrm{s}$; nongymnasts: $M=12.85 \mathrm{~mm} / \mathrm{s}, S D=5.25 \mathrm{~mm} / \mathrm{s}$; $p=.57$ ), or area, $t(14)=0.2$ (gymnasts: $M=120.95 \mathrm{~mm}^{2}$, $S D=18.95 \mathrm{~mm}^{2}$; nongymnasts: $M=129.73 \mathrm{~mm}^{2}, S D=$ $20.7 \mathrm{~mm}^{2} ; p=.60$ ), of the COP between the two groups' performances during the 30 -s trials in quiet stance with the weight. The two groups displayed similar patterns of the COP displacements in the anterior-posterior axis. All participants' responses to perturbations were organized with a destabilization phase at weight removal, preceding a restabilization phase and then a final return to stable postural state (see Figure 2).

\section{Duration of the Sway Latencies and Magnitude of the COP Displacements}

The SLs were significantly, $t(14)=-4.66, p<.01$, shorter for the gymnasts $(M=4.03 \mathrm{~s}, S D=0.82 \mathrm{~s})$ than for the nongymnasts $(M=6.29 \mathrm{~s}, S D=1.09 \mathrm{~s})$. However, no significant difference was observed for either (a) the proportion of SL devoted to the destabilization phase, $t(14)=-.32, p=.75$, between the gymnasts $(M=9.68 \%)$ and the nongymnasts $(M$ $=10.17 \%$ ) or (b) the proportion of SL, $t(14)=0.32, p=.75$, devoted to the restabilization phase $(M=90.32 \%$ and $89.83 \%$ for the gymnasts and nongymnasts, respectively).

The maximal magnitude of the COP displacements for the gymnasts was $M=115.49 \mathrm{~mm}, S D=16.88 \mathrm{~mm}$, and for the nongymnasts it was $M=128.30 \mathrm{~mm}, S D=24.18 \mathrm{~mm}$. COP displacement during the SL phase did not significantly differ between groups, $t(14)=-1.23, p=.24$.

\section{SE of the COP Displacements}

We observed a significant, $F(1,28)=418.18, p<.01$, phase effect but noted neither a significant expertise effect, $F(1,14)=551, p=.37$, nor a significant interaction effect, $F(1,28)=2.61, p=.09$.

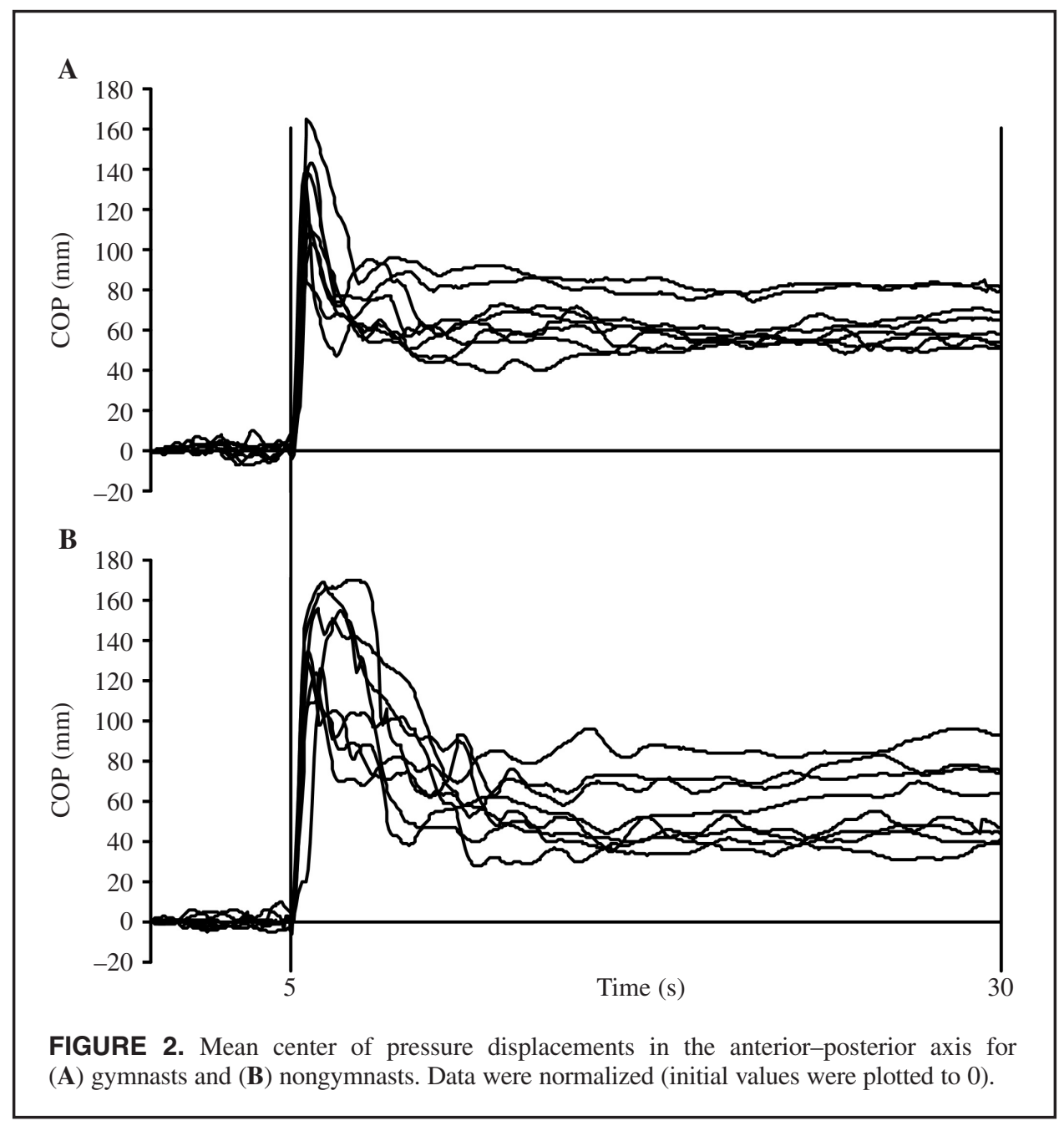


The post hoc tests showed that the SEs of the COP displacements were significantly larger during the destabilization phase $(M=10.59, S D=1.94)$ than during either the restabilization phase $(M=1.17, S D=0.38 ; p<.01)$ or the stable phase $(M=0.22, S D=0.06 ; p<.01)$. But no significant difference ( $p=.06$ ) was observed between the SE of the COP displacements during the restabilization and stable phases.

\section{Joint Angle Analysis}

Table 2 shows the SE and MAVM data of the three angles, and Figure 3 illustrates that data for the two groups.

From a descriptive point of view (Figure 4), we observed a knee-ankle coordination mode for the gymnasts group during the destabilization phase, whereas we observed a hip-ankle mode for the nongymnasts group. A hip-ankle coordination mode was observed for the two groups during the restabilization phase, but the magnitude of the joint movements appeared to be larger for the nongymnasts than for the gymnasts. Last, during the stable phase, both groups showed limited angular movements.

Regardless of which joint we considered, we observed (a) a significant expertise effect- $F(1,14)=8.27 ; F(1,14)$ $=63.10 ; F(1,14)=59.18$ - for the ankle, knee, and hip, respectively, and (b) a significant phase effect $-F(1,28)$ $=863.04 ; F(1,28)=662.33 ; F(1,28)=632.78$-for the ankle, knee, and hip, respectively $(p<.01)$.

Moreover, we also noted a significant interaction effect for the ankle, $F(1,28)=43.84$; knee, $F(1,28)=69.58$; and hip, $F(1,28)=24.25$; joint angles $(p<.01)$.

The post hoc tests showed that for both gymnasts and nongymnasts, the SEs of the ankle, knee, and hip joints were significantly larger $(p<.01)$ during destabilization than during restabilization and significantly larger $(p<$ .01) during destabilization than during the stable phase. Moreover, between the restabilization phase and the stable phase, the SEs of the angles were not significantly different $(p>.05)$, except for the ankles and hips of the nongymnasts group, for which the SEs were significantly larger $(p<.01)$ during the restabilization phase.
During the destabilization phase, the SE was significantly larger $(p<.01)$ for the gymnasts group than for the nongymnasts group for both the ankle and knee angles. In contrast, the SEs for the hip angles were significantly larger $(p<.01)$ for the nongymnasts than for the gymnasts.

During the restabilization phase, the SEs were significantly larger for the nongymnasts group than for the gymnasts group for both the ankle angles $(p=.04)$ and the hip angles $(p=.04)$. For the knee angles, we observed no significant difference $(p=.93)$. During the final stable phase, the SEs of the joint angle of the gymnasts and nongymnasts were not significantly different, regardless of which joint we considered $(p>.99)$.

\section{Maximal Angle Variation Magnitude}

The effect of expertise on the MAVM was significant for all angles, $F(1,14)=29.94 ; F(1,14)=78.08 ; F(1,14)$ $=194.56$, for the ankles, knees, and hips, respectively ( $p$ $<.001)$. Moreover, the effect of phase on the MAVM was also significant for all joints, $F(1,28)=274.25 ; F(1,28)=$ $184.37 ; F(1,28)=239.02$, for the ankles, knees, and hips, respectively $(p<.01)$. Last, we noted interaction effects for the three joints, $F(1,28)=51.69 ; \mathrm{F}(1,28)=56.60 ; \mathrm{F}(1,28)=$ 70.52 , for the ankles, knees, and hips, respectively $(p<.01)$.

The post hoc tests showed that the MAVM of the ankles, knees, and hips were significantly larger during the destabilization phase than during the restabilization phase $(p<.01$ for both the gymnasts and nongymnasts), except for the nongymnasts' ankles, for which we observed no significant difference $(p=.82)$. Moreover, the MAVM during the destabilization phase was significantly larger than during the stable phase for both the nongymnasts and the gymnasts $(p<.01)$. Last, no significant differences were observed between the MAVM during the restabilization phase and the MAVM during the stable phase $(p>.05)$, except for the MAVM for the ankles and hips of the nongymnasts group, which were significantly larger $(p<.01)$ during the restabilization phase.

The post hoc tests showed that during the destabilization phase, the MAVMs were significantly larger for the gymnasts

TABLE 2. Means and Standard Deviations of Standard Error and Maximal Angle Variation Magnitude of Destabilization, Restabilization, and Stable Phases for Gymnasts and Nongymnasts

\begin{tabular}{|c|c|c|c|c|c|c|c|c|c|c|c|c|}
\hline \multirow[b]{3}{*}{ Variable } & \multicolumn{6}{|c|}{ Gymnasts } & \multicolumn{6}{|c|}{ Nongymnasts } \\
\hline & \multicolumn{2}{|c|}{ Ankles } & \multicolumn{2}{|c|}{ Knees } & \multicolumn{2}{|c|}{ Hips } & \multicolumn{2}{|c|}{ Ankles } & \multicolumn{2}{|c|}{ Knees } & \multicolumn{2}{|c|}{ Hips } \\
\hline & $M$ & $S D$ & $M$ & $S D$ & $M$ & $S D$ & $M$ & $S D$ & $M$ & $S D$ & $M$ & $S D$ \\
\hline \multicolumn{13}{|c|}{ Standard error angle } \\
\hline Destabilization & 1.36 & 0.16 & 1.49 & 0.19 & 0.97 & 0.16 & 0.99 & 0.1 & 0.7 & 0.14 & 1.5 & 0.13 \\
\hline Restabilization & 0.2 & 0.06 & 0.19 & 0.06 & 0.17 & 0.05 & 0.32 & 0.03 & 0.16 & 0.04 & 0.31 & 0.05 \\
\hline Stable state & 0.15 & 0.02 & 0.14 & 0.03 & 0.16 & 0.15 & 0.16 & 0.02 & 0.11 & 0.04 & 0.16 & 0.03 \\
\hline \multicolumn{13}{|c|}{ Maximal angle variation magnitude } \\
\hline Destabilization & 32.64 & 5.14 & 38.15 & 4.74 & 12.52 & 4.14 & 20.9 & 3.32 & 9.99 & 4.48 & 43.77 & 6.1 \\
\hline Restabilization & 6.44 & 1.71 & 5.16 & 4.27 & 5.12 & 4.07 & 9.96 & 2.18 & 6.9 & 3.79 & 26.51 & 6.25 \\
\hline Stable state & 2.16 & 0.4 & 1.74 & 0.71 & 2.21 & 0.38 & 2.07 & 0.71 & 1.16 & 0.75 & 2.01 & 0.57 \\
\hline
\end{tabular}




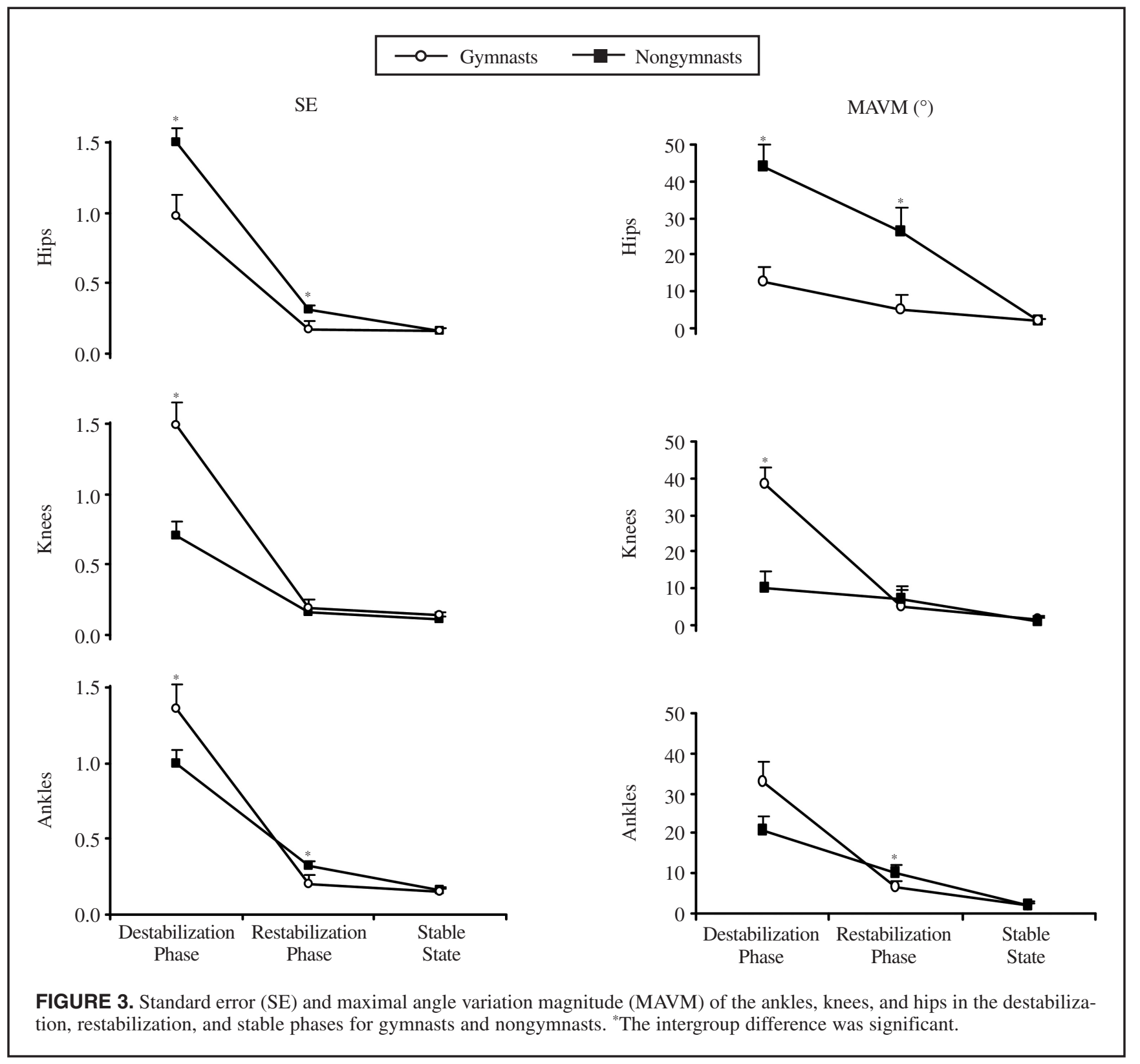

than for the nongymnasts when we considered the knee angles $(p<.01)$. In contrast, they were significantly larger for the nongymnasts than for the gymnasts when the hip angles were considered $(p<.01)$. No significant difference between groups was observed for the ankle angles $(p=.27)$.

During the restabilization phase, the MAVMs were significantly larger for the nongymnasts group than for the gymnasts group when we considered the ankles and hips $(p<.01)$, but no difference was observed for the knee angles $(p=.99)$.

During the final stable phase, the MAVM of the angles of the gymnasts and nongymnasts was not significantly different, regardless of which joint we considered $(p>.05)$.

\section{Discussion}

The aim of this study was to determine the influence of expertise in gymnastics on postural control. First, our results showed that gymnasts and nongymnasts were able to maintain the same stable postural state, with or without a weight pulling on their backs. Thus, the measurement of performance (such as COP area and velocity) in a stable-stance maintenance task is not sensitive enough to identify the impact of gymnastics expertise on postural characteristics (Asseman et al., 2004; Balter et al., 2004; Vuillerme, Danion, et al., 2001; Vuillerme \& Nougier, 2004). Therefore, we added specific demands by modifying the constraints to their posture. This required postural adaptations from which postural control modes emerged (Bardy et al., 1999; Riccio \& Stoffregen, 1988; Stoffregen \& Riccio, 1988; Stoffregen et al., 1999). As illustrated in Figure 4, the gymnasts and nongymnasts used a different postural coordination mode. The difference in the use of the joints, as the COP oscillations, depended on the participant's prior experience. 


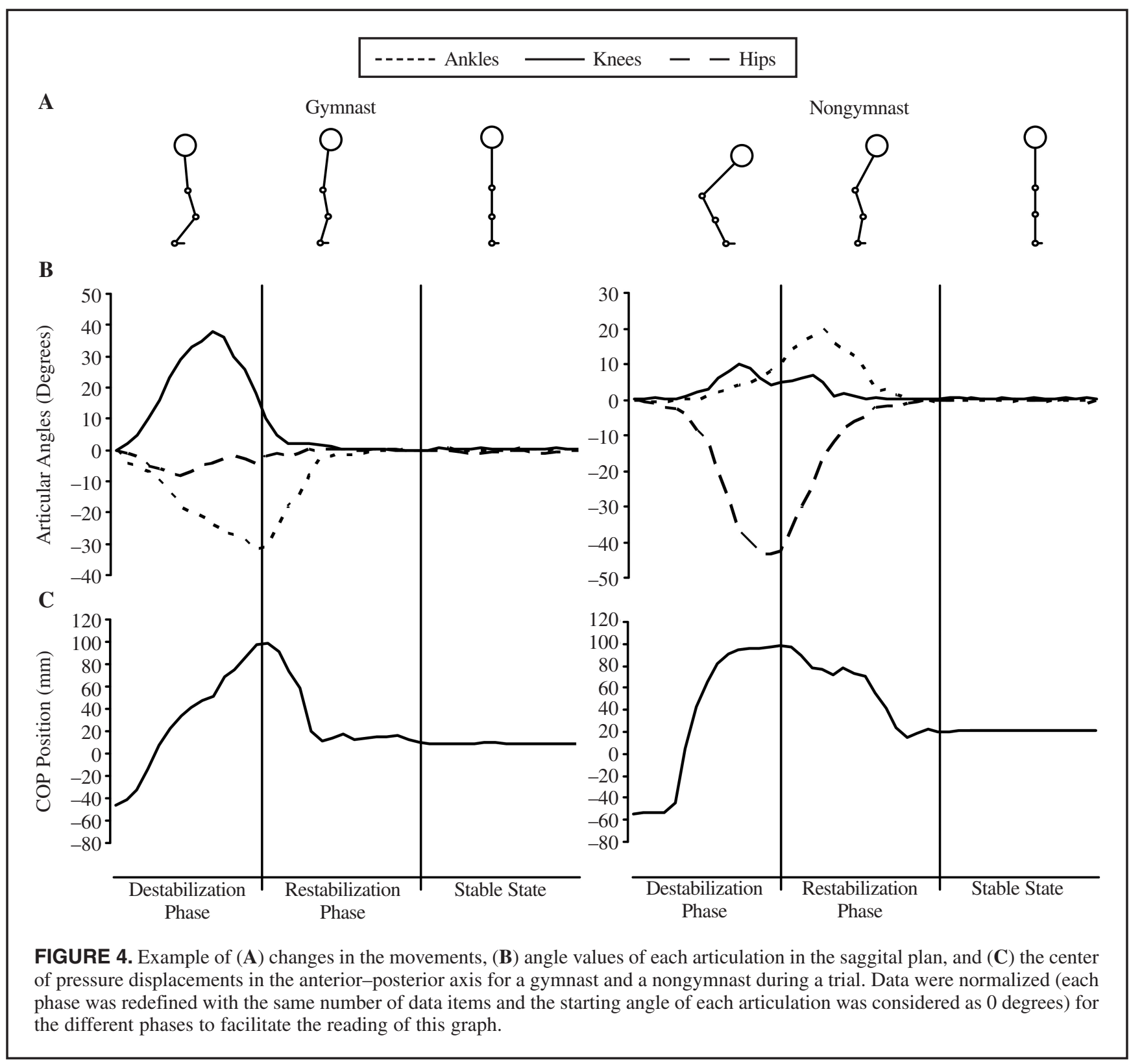

From a temporal point of view, the SLs showed that the gymnasts were able to rapidly recover a stable postural state and decrease their COP variations, in contrast to the control group. As we noted in the introduction, an individual attains postural stability when uncontrolled movements in the perception-action coupling, which hinder suprapostural task performance, are minimized (Riccio \& Stoffregen, 1988; Stoffregen et al., 1999). The gymnasts seemed to be able to compensate for destabilization more rapidly to accomplish the suprapostural task (looking at the target in front of them); this behavior is coherent with the development of a postural adaptation that was relevant to the constraints.

The gymnasts in fact were able to start the restabilization phase after only $0.39 \mathrm{~s}-$ nearly twice as fast as the time of the nongymnasts. Moreover, they seemed more able to con- trol their body movements than the nongymnasts. A higher sensitivity to body movement develops with gymnastics experience (Bringoux et al., 2000; Danion, Boyadjian, \& Marin, 2000; Vuillerme, Teasdale, et al., 2001). The gymnasts were able to take advantage of changes in body acceleration relative to gravito-inertial force, which provided hints about their postural instability (Stoffregen \& Riccio, 1988), more quickly than the nongymnasts. Our results could be interpreted as an optimization in perception-action coupling with expertise. Recently, Le Runigo, Benguigui, and Bardy (2005) made a similar interpretation in a study on the effects of tennis practice on interceptive actions.

In the present study, the greater sensitivity to the body movements are not explicable by an improvement in the sensory systems, such as the vestibular receptors (Balter et al., 2004). Thus, the gymnasts probably had developed 
a better ability to exploit multimodal information (Stoffregen \& Bardy, 2001; Van der Kooij et al., 1999). Assuming that gymnastics requires the stabilization of stance after complex and unusual movements, the difference in the gymnasts' and nongymnasts' reactions to body movements seems to have resulted from a modification in the gymnasts' sensitivity or the ability to exploit the information emerging from multimodal perception. This possibility is consistent with previous findings on ballet dancers and track athletes, who exhibited different dynamic patterns of postural sway relative to their practice and the multimodal situation (Schmit, Regis, \& Riley, 2005).

Previous findings and our own results have suggested differences at the level of the integrative mechanisms involved in postural regulation. The nature of the cortical involvement in postural responses is known to depend on prior experience, and the organism may provide both speed and flexibility for modifying environmentally appropriate responses to a loss of balance (Jacobs \& Horak, in press). The cortical plasticity, with the gymnastics experience, may result in a functional reorganization of motor behavior so that it becomes more reactive. This possibility also suggests the specific flexibility of coordination associated with this cortical adaptation. Yet, our gymnasts and nongymnasts showed similar kinetics organizations in the transition from stable postural states with weight to those without weight. For both groups, the COP magnitudes in the destabilization phase were similar, and a progressive decrease in oscillations followed. Moreover, the same relative proportion of SLs was devoted to the destabilization phase and the restabilization phase. This suggests that changes with experience influenced only the reaction time. However, the angular variations and magnitudes of the two groups appeared to be different (see Figure 4).

The kinematics results showed a significant difference between the gymnasts and nongymnasts in the destabilization phase for the ankle, knee, and hip variations and in the restabilization phase for the ankle and hip variations. The experts in gymnastics displayed more angular movements (variations and magnitudes) in the destabilization phase and then more rapidly decreased their angular variations. For the nongymnasts, angular movements were lower in the destabilization phase and higher in the restabilization phase. They produced a less emphatic angular reaction but took more time than the gymnasts to recover a stable angular state. The gymnasts use a different organization of the postural control to react to the destabilization. Consequently, they demonstrated a specific flexibility in their postural responses (Jacobs \& Horak, in press). More precisely, the postural synergies used to regulate balance were specific to each group (see Figure 4). The nongymnasts primarily used their hips when confronted with perturbation because, according to Horack and Nashner (1986), mobilizing the hip joints allows for rapid repositioning of the center of gravity to guarantee equilibrium. In contrast, the gymnasts preferred to use their knees more than their hips. Thus, it appears that expertise in gymnastics leads to the development of specific coordination modes. The constraints imposed by the difficulty of maintaining stable balance after each acrobatic element and also the rules that penalize hip movements in gymnastics (Fédération Internationale de Gymnastique, 2005) could enable us to explain this phenomenon. In a study of the ankle-hip coordination phase relation in expert gymnasts, Marin et al. (1999) made a similar interpretation.

Previous researchers have demonstrated that knee movement rapidly lowers the center of gravity in the vertical axis, decreasing the height of the body mass relative to the support surface (Nashner \& McCollum, 1985). Yet in the present experiment, the destabilization was in the anterior-posterior axis. This finding suggests that the knees also contribute to anterior-posterior regulation. Indeed, lowering the center of mass allows the individual to decrease the anterior-posterior axis of rotation and hence the moment of inertia. Moreover, the knee movements were associated with hip and ankle movements. Thus, rather than describing postural control as the result of dissociated strategies (Nashner \& McCollum), we suggest that postural coordination emerges from the interaction among constraints of the environment, the task, and the organism (Bardy et al., 1999; Riccio \& Stoffregen, 1988; Stoffregen \& Riccio, 1988). The organism adapts postural coordination, according to the properties of the environment and the organism, to facilitate the accomplishment of suprapostural tasks (Stoffregen et al., 1999). In the present experiment, the suprapostural task was to visually focus on a target without any instructions on which postural control to adopt. The two groups with different prior experience produced their own adaptations to the constraints (see Figure 4). To maintain balance while looking at the target, the gymnasts and nongymnasts used different postural patterns regarding the reaction to body movements and the coordination mode involved in the postural-suprapostural task. Thus, the present results suggest that gymnastics expertise led to a modification in the postural pattern used to facilitate the performance of a suprapostural task.

In conclusion, this study illustrated the influence of experience, particularly gymnastics expertise, on postural control. Our results showed an ambiguous relation between the consequences of kinetics and kinematics relative to the perturbation. The results suggest that they were complementary but not similar, providing a full analysis of the postural control. Thus, our results are congruent with the idea that experts in sports requiring fine perceptive-motor control develop a shorter sensory-motor delay (Le Runigo et al., 2005). Gymnasts develop specific postural flexibility and adapt differently to constraints. Our specific experiences appear to indicate an additional constraint on the postural action possibilities, not only in specific activities but also in nonspecific postural suprapostural tasks. Differences in the emergence of stability control depend on prior experience. Gymnastics, in terms of reactions and coordination, 
modifies postural control. The present study suggests that perceptive-motor experience leads to a functional modification of postural control. Future researchers should conduct more studies to specify the influence of experience in the perception-action coupling.

\section{ACKNOWLEDGMENTS}

The authors thank the two anonymous reviewers for their valuables comments. This study was supported by the French Ministère de l'Enseignement Supérieur et de la Recherche, grant $13426-2004$

\section{REFERENCES}

Asseman, F., Caron, O., \& Cremieux, J. (2004). Is there a transfer of postural ability from specific to unspecific postures in elite gymnasts? Neuroscience Letters, 358(2), 83-86.

Balter, S. G., Stokroos, R. J., Akkermans, E., \& Kingma, H. (2004). Habituation to galvanic vestibular stimulation for analysis of postural control abilities in gymnasts. Neuroscience Letters, 366(1), 71-75.

Bardy, B. G., Marin, L., Stoffregen, T. A., \& Bootsma, R. J. (1999). Postural coordination modes considered as emergent phenomena. Journal of Experimental Psychology Human Perception and Performance, 25, 1284-1301.

Bringoux, L., Marin, L., Nougier, V., Barraud, P. A., \& Raphel, C. (2000). Effects of gymnastics expertise on the perception of body orientation in the pitch dimension. Journal of Vestibular Research, 10, 251-258.

Bronstein, A. M., \& Guerraz, M. (1999). Visual-vestibular control of posture and gait: Physiological mechanisms and disorders. Current Opinion in Neurology, 12(1), 5-11.

Clark, S., \& Riley, M. A. (2007). Multisensory information for postural control: Sway-referencing gain shapes center of pressure variability and temporal dynamics. Experimental Brain Research, 176, 299-310.

Danion, F., Boyadjian, A., \& Marin, L. (2000). Control of locomotion in expert gymnasts in the absence of vision. Journal of Sports Sciences, 18, 809-814.

Duarte, M., \& Zatsiorsky, V. M. (2000). On the fractal properties of natural human standing. Neuroscience Letters, 283, 173-176.

Era, P., Konttinen, N., Mehto, P., Saarela, P., \& Lyytinen, H. (1996). Postural stability and skilled performance: A study on top-level and naive rifle shooters. Journal of Biomechanics, 29, 301-306.

Fédération Internationale de Gymnastique. (2005). Code of point. Freiburg, Germany: Author.

Gibson, J. J. (1986). The ecological approach to visual perception. Hillsdale, NJ: Erlbaum.

Golomer, E., Cremieux, J., Dupui, P., Isableu, B., \& Ohlmann, T. (1999). Visual contribution to self-induced body sway frequencies and visual perception of male professional dancers. Neuroscience Letters, 267, 189-192.

Horak, F. B. (2006). Postural orientation and equilibrium: What do we need to know about neural control of balance to prevent falls? Age and Ageing, 35, 7-11.

Horak, F. B., \& Nashner, L. M. (1986). Central programming of postural movements: Adaptation to altered support-surface configurations. Journal of Neurophysiology, 55, 1369-1381.

Jacobs, J. V., \& Horak, F. B. (in press). Cortical control of postural responses. Journal of Neural Transmission, 114, 1339-1348.
Kluzik, J., Horak, F. B., \& Peterka, R. J. (2005). Differences in preferred reference frames for postural orientation shown by aftereffects of stance on an inclined surface. Experimental Brain Research, 162, 474-489.

Le Runigo, C., Benguigui, N., \& Bardy, B. G. (2005). Perceptionaction coupling and expertise in interceptive actions. Human Movement Science, 24, 429-445.

Lejeune, L., Anderson, D. I., Campos J. J., Witherington, D. C., Uchiyama, I., \& Barbu-Roth, M. (2006). Responsiveness to terrestrial optic flow in infancy: Does locomotor experience play a role? Human Movement Science, 25(1), 4-17.

Marin, L., Bardy, B. G., \& Bootsma, R. J. (1999). Level of gymnastic skill as an intrinsic constraint on postural coordination. Journal of Sports Sciences, 17, 615-626.

Mesure, S., Amblard, B., \& Cremieux, J. (1997). Effect of physical training on head-hip co-ordinated movements during unperturbed stance. Neuroreport, 8, 3507-3512.

Nashner, L. M., \& McCollum, G. (1985). The organization of human postural movements: A formal basis and experimental synthesis. Behavioral and Brain Science, 8, 135-172.

Perrin, P., Deviterne, D., Hugel, F., \& Perrot, C. (2002). Judo, better than dance, develops sensorimotor adaptabilities involved in balance control. Gait and Posture, 15, 187-194.

Riccio, G. E., \& Stoffregen, T. A. (1988). Affordances as constraints on the control of stance. Human Movement Science, 7 , 265-300.

Riley, M. A., \& Clark, S. (2003). Recurrence analysis of human postural sway during the sensory organization test. Neuroscience Letters, 342(1-2), 45-48.

Sarre, F. (2000). Reconstructing the archetype: Initial bipedalism as a realistic model for vertebrate evolution. Bipedia, 18, 1-11.

Schmit, J. M., Regis, D. I., \& Riley, M. A. (2005). Dynamic patterns of postural sway in ballet dancers and track athletes. Experimental Brain Research, 163(3), 370-378.

Sparrow, W. A., \& Tirosh, O. (2005). Gait determination: A review of experimental methods and the effects of ageing and gait pathologies. Gait and Posture, 22, 362-371.

Stoffregen, T. A., \& Bardy, B. G. (2001). On specification and the senses. Behavioral and Brain Science, 24, 195-261.

Stoffregen, T. A., \& Riccio, G. E. (1988). An ecological theory of orientation and the vestibular system. Psychological Revue, 95(1), 3-14.

Stoffregen, T. A., Smart, L. J., Bardy, B. G., \& Pagulayan, R. J. (1999). Postural stabilisation of looking. Journal of Experimental Psychology Human Perception and Performance, 25, $1641-1658$.

Van der Kooij, H., Jacobs, R., Koopman, B., \& Grootenboer, H. (1999). A multisensory integration model of human stance control. Biological Cybernetics, 80, 299-308.

Vuillerme, N., Danion, F., Marin, L., Boyadjian, A., Prieur, J. M., Weise, I., et al. (2001). The effect of expertise in gymnastics on postural control. Neuroscience Letters, 303(2), 83-86.

Vuillerme, N., \& Nougier, V. (2004). Attentional demand for regulating postural sway: The effect of expertise in gymnastics. Brain Research Bulletin, 63(2), 161-165.

Vuillerme, N., Teasdale, N., \& Nougier, V. (2001). The effect of expertise in gymnastics on proprioceptive sensory integration in human subjects. Neuroscience Letters, 311(2), 73-76.

Submitted December 4, 2006

Revised May 29, 2007

Second revision September 6, 2007 
Copyright of Journal of Motor Behavior is the property of Heldref Publications and its content may not be copied or emailed to multiple sites or posted to a listserv without the copyright holder's express written permission. However, users may print, download, or email articles for individual use. 